\title{
Effects of Intensity-Specific Acute Exercise on Paired-Associative Memory and Memory Interference
}

\author{
Lindsay Crawford and Paul Loprinzi * \\ Exercise \& Memory Laboratory, Department of Health, Exercise Science and Recreation Management, \\ The University of Mississippi, Oxford, MS 38677, USA; lcrawfor@go.olemiss.edu \\ * Correspondence: pdloprin@olemiss.edu
}

Received: 10 April 2019; Accepted: 22 May 2019; Published: 25 May 2019

\begin{abstract}
The improvement of memory performance is an ever-growing interest in research, with implications in many fields. Thus, identifying strategies to enhance memory and attenuate memory interference is of great public health and personal interest. The objective of this paper was to evaluate the role of intensity-specific acute exercise on improving paired-associative memory function and attenuating memory interference. A counterbalanced, randomized controlled, within-subject experimental design was employed. The three counterbalanced visits included a control visit, moderate-intensity exercise (50\% of HRR; heart rate reserve) and vigorous-intensity exercise ( $80 \%$ of HRR), all of which occurred prior to the memory assessment. To evaluate memory interference, an $\mathrm{AB} / \mathrm{AC}$ paired-associative task was implemented for each laboratory visit. The number of correctly recalled words from List 1 (AB-DE) was statistically significantly $\left(\mathrm{F}=4.63, p=0.01, \eta^{2} \mathrm{p}=0.205\right)$ higher for the vigorous-intensity condition $(\mathrm{M}=6.53, \mathrm{SD}=1.54)$ as compared to moderate-intensity $(\mathrm{M}=6.11, \mathrm{SD}=1.59)$ and control $(\mathrm{M}=5.00, \mathrm{SD}=2.56)$ conditions. No statistical significance was found between proactive interference or retroactive interference across the experimental conditions. This experiment provides evidence for an intensity-specific effect of acute exercise on short-term, paired-associative memory, but not memory interference.
\end{abstract}

Keywords: $\mathrm{AB} / \mathrm{AC}$ paradigm; memory; paired associates; exercise; memory interference; intensity-specific; proactive interference; retroactive interference; moderate intensity; vigorous intensity

\section{Introduction}

Memory function is, unquestionably, critical for optimal daily function and is associated with quality of life and mortality [1]. Thus, identifying factors that influence memory function is of great public health and personal interest. Various brain structures, such as the hippocampus and prefrontal cortex, play critical roles in influencing memory function [2,3]. In particular, these respective brain structures subserve episodic and working memory capacity. Episodic memory function refers to the retrospective recall of information from a spatial-temporal context [4], whereas working memory involves the transient storage of information while concurrently processing competing stimuli [5].

Acute and chronic exercise have been shown to enhance episodic memory [6-19]. Notably, however, as we have demonstrated in a recent systematic review [20], there may be an intensity-specific effect of acute exercise on enhancing episodic memory, with higher-intensity exercise potentially being optimal. Further, as we thoroughly addressed elsewhere [20,21], higher-intensity exercise may enhance long-term potentiation (LTP) to a greater extent than lower-intensity exercise, which may explain these potential intensity-specific effects, as LTP is considered an underlying mechanistic correlate of episodic memory [22]. However, as we discussed recently [20], higher-intensity acute exercise may impair working memory by increasing the levels of dopamine and norepinephrine in the prefrontal cortex and, ultimately, dampen neural activity via cAMP opening of nearby K+ channels [20,23]. 
There are a limited number of within-subject experimental studies evaluating whether memory is differentially affected by varying acute exercise intensities. Further, within this field, relatively few studies have evaluated the effects of acute exercise on paired-associative learning, a hippocampal-dependent task [24]. Paired-associative learning is a classic memory paradigm used to understand how individuals encode and retrieve newly learned associations among stimuli. Thus, it is an important task used to examine and understand the mechanisms of learning and forgetting. To address this gap in the literature, an objective of the present experiment was to evaluate whether there is an optimal acute exercise intensity to enhance short-term memory, as assessed from a paired-associative task. We hypothesize that higher-intensity acute exercise will be most advantageous in improving short-term, paired-associative memory.

To further complement this body of literature, another objective of this experiment was to evaluate whether acute exercise can attenuate memory interference, which can also be assessed from a paired-associative learning task. Memory interference is one of the many forces behind the impairment of memory retention [25]. There are two common types of memory interference, proactive interference and retroactive interference [26]. Proactive interference occurs when older information interferes with the recall of newly attained information. Retroactive interference occurs in the opposite direction; new information disrupts the recall of older information. As with most aspects of memory, interference may be influenced by several factors including gender, age, cognitive capacity, and content similarity [27-30]. Regarding the latter, if information is similar (e.g., listing names of neuronal pathways), interference is more likely to occur [31].

Physical activity is associated with increased prefrontal cortex (PFC) and hippocampal volume along with increased levels of brain-derived neurotrophic factor (BDNF) [29,32]. Acute exercise-induced BDNF release is associated with improved medial temporal lobe function, which is linked with cognitive enhancement, especially when exercise occurs in an enriched environment [32]. The PFC and hippocampus are stimulated during exercise, increasing neuronal excitability, priming the cells into encoding particular stimuli, thereby facilitating memory formation [33]. These same regions are also utilized during memory encoding, retrieval, and, importantly, distinguishing target information from similarly related information (e.g., paired-associate tasks) [29,34]. The hippocampus is not only utilized during encoding, but plays an important role in pattern separation, while exercise has been demonstrated to increase hippocampal activity, which in turn, may help to facilitate hippocampal-induced pattern separation [35]. Other mechanisms, related to pattern separation (i.e., attenuate a memory interference effect) that may be influenced by exercise, include increased amygdala-hippocampal functional connectivity, neurogenesis, and dentate gyrus activation within the hippocampus [36,37]. All of these networks provide potential explanations of exercise benefiting paired-associative learning and attenuating memory interference. Another explanation of this exercise-memory interaction, but from a cognitive psychology perspective, is the cognitive-energetic approach [23]. This multidimensional model suggests that exercise-related arousal and activation, coordinated by cognitive effort, have a voluntary control on psychological attention [23]. This effort-guided psychological attention, which is likely to be moderated by exercise intensity, may play a critical role in memory encoding and, ultimately, paired-associative learning [23].

Further, moderate- and vigorous-intensity exercise have been shown to induce mixed effects on memory enhancement [38], highlighting the importance of additional investigations on this topic. Higher-intensity exercise may decrease oxygenation of the prefrontal cortex, increase the levels of norepinephrine and dopamine in the PFC, which, as mentioned, may weaken neuronal activity, ultimately impairing PFC function [20,39]. Others, however, have demonstrated that acute vigorous exercise and moderate-intensity exercise prior to encoding are effective at improving working memory and enhancing PFC function [20]. These conflicting results may be due to many factors, including the subject's fitness level, implementation of novel environments, and the modality of exercise. 
As stated, there is a dearth of research investigating the intensity-specific effects of acute exercise on paired-associative learning and memory interference. Further, what has yet to be investigated is whether acute exercise can minimize a memory interference effect, as assessed via the AB-DE AC-FG paradigm. Such an effect is plausible given the observed effect that acute exercise has on post-exercise neuronal activity in the prefrontal cortex [40], coupled with the effect the prefrontal cortex plays in minimizing a memory interference effect [34]. Thus, a key focus of this novel study was to examine whether acute exercise can reduce proactive and retroactive memory interference, when the acute bout of exercise occurs prior to the memory task. This time period of exercise, occurring prior to the memory task, is in contrast to related consolidation studies that have evaluated whether exercise, when occurring during the consolidation period, can enhance memory [41,42]. Unlike these studies, the aim of the present complementary approach was to examine the effects of exercise on cognitive processes underlying resolution of associative interference (proactive and retroactive). We hypothesize that acute exercise, particularly higher-intensity exercise [20], will enhance short term, paired-associative memory and attenuate a memory interference effect.

\section{Methods}

\subsection{Study Design}

All data collection occurred between August and October of 2018, in the authors' Exercise and Memory Laboratory. A total of 19 participants completed three laboratory visits (at approximately the same time of day for each visit; e.g., each visit occurring at approximately 11:00), with these visits occurring at least $24 \mathrm{~h}$ apart. This was based on a power analysis, utilizing $\mathrm{G}^{*}$ Power, indicating that 18 participants would be needed to achieve adequate statistical power, with inputs of an $\alpha$ error probability of $0.05,1-\beta$ error probability of $0.85,3$ within-subject measurements, 0.5 correlation among repeated measures, and an effect size $\mathrm{F}$ of 0.33 .

A counterbalanced, randomized controlled, within-subject design was employed. The three counterbalanced visits included a control visit, moderate-intensity exercise (50\% of HRR; heart rate reserve) and vigorous-intensity exercise ( $80 \%$ of HRR).

\subsection{Participants}

Participants provided voluntary consent prior to participation. This project was approved by the ethics committee at the University of Mississippi (\#19-004). Participants included undergraduate and graduate students of the University of Mississippi. The sample originally included 20 participants, but one participant was excluded due to the inability to exercise based on responses from the PAR-Q. The final sample included 19 participants (6 males and 13 females from the ages of 18 to 35 years). This sample size is consistent with other work in this area $[43,44]$. Participants were excluded if they: self-reported as a daily smoker [45,46], self-reported being pregnant [47], exercise within 5 hours of testing [48], consumed caffeine within 3 hours of testing [49], had a concussion or head trauma within the past 30 days [50], had a diagnosis of ADHD [51], used marijuana or other illegal drugs within the past 30 days [52], or were considered a daily alcohol user ( $>30$ drinks/month for women; $>60$ drinks/month for men) [53]. These exclusionary criteria were employed to minimize a potential confounding effect on memory. Participant handedness was not measured for this experiment. For a schematic of the experimental protocol, see Figure 1. 


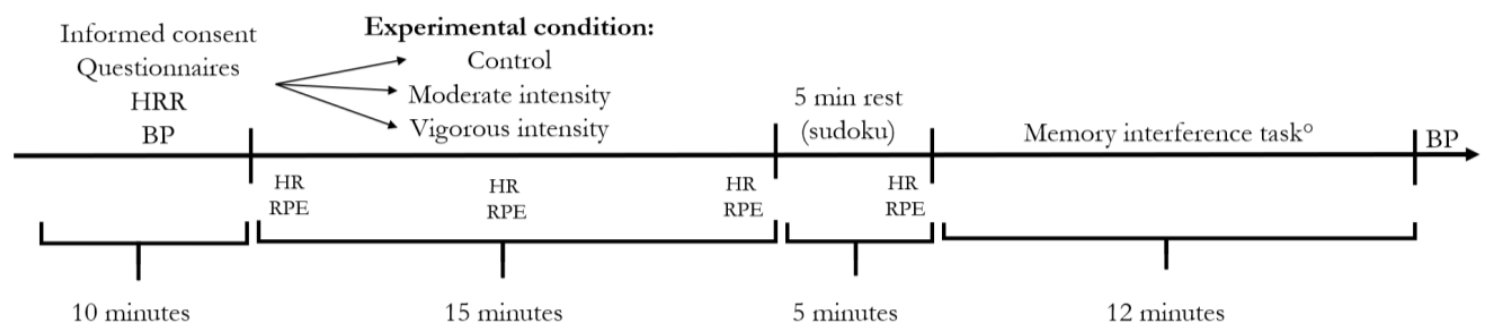

Figure 1. Schematic of the experimental protocol (minimum of $24 \mathrm{~h}$ follow-up between each experimental condition). $\mathrm{HRR}=$ heart rate reserve; $\mathrm{BP}=$ blood pressure; $\mathrm{RPE}=$ rate of perceived exertion see Table 1.

Table 1. Example protocol for evaluating memory interference.



\subsection{Experimental Conditions}

Each of the three experimental conditions occurred prior to the memory task. The active control condition, similar to other studies [41], involved playing a medium-level, on-line administered, Sudoku puzzle. Participants in this control condition completed this time-matched task for $20 \mathrm{~min}$ prior to completing the memory task (described below). The website for this puzzle is located here: https://www.websudoku.com/.

The two exercise conditions (moderate-intensity and vigorous-intensity) included a 15-min bout of treadmill exercise (brisk walking and jogging, respectively), followed by a 5-min recovery period. Previous literature has demonstrated that this exercise mode and a 15-min bout length is sufficient in demonstrating exercise-induced improvements in memory $[13,14,20,43,54-56]$. The HRR equation used to evaluate exercise intensity was: $\mathrm{HRR}=[(\mathrm{HR} \max -\mathrm{HRrest}) \times \%$ intensity $]+$ HRrest. To calculate HRrest, at the beginning of the visit, participants sat quietly for 5 minutes, and HR was recorded from a chest-worn Polar HR monitor. To estimate HRmax, we calculated the participants estimated HRmax from the formula, 220-age [57]. Utilizing the HRR method, the moderate-intensity and vigorous-intensity exercise visits, respectively $50 \%$ and $80 \%$, were entered into the above formula. These respective intensities represent moderate- and vigorous-intensity exercise [58].

Heart rate was measured for each experimental condition at rest, halfway through the bout of exercise $(7.5 \mathrm{~min})$, at the end of the bout $(15 \mathrm{~min})$, and after a 5 -min rest period. 


\subsection{Memory}

In research settings, memory interference is often measured using a paired-associative task-tasks that include pairs of objects (e.g., pictures, words), which are to be memorized and subsequently recalled [59-61]. For this experiment, the AB-DE AC-FG paired-associative paradigm was utilized. This method was chosen due to its ability to create an interference effect and measure proactive interference and retroactive interference simultaneously. This model is very similar to an $A B / A C$ paradigm, but it contains control word pairs. The AB-DE AC-FG method involves exposure to, and the recall of, two individual word lists containing unrelated words. This task has previously been shown to provide evidence of construct validity [62]. Other related tasks of memory interference have demonstrated evidence of reliability [63]. List 1 is comprised of AB-DE word pairs while List 2 is comprised of AC-FG word pairs. Each list includes multiple word pairs, half being interference pairs $(\mathrm{AB}, \mathrm{AC})$ and half being control pairs (DE, FG). The " $\mathrm{A}$ " words being the only ones which repeat (e.g., $A B$ "sugar" - temple, $A C$ "sugar" — canoe), causing memory interference, while the remainder of the lists are non-repeating words. As stated previously, the modified-modified free recall (MMFR) list is similar to the recall of $\mathrm{AB}, \mathrm{AC}$ words in an $\mathrm{AB} / \mathrm{AC}$ paradigm, but this particular method includes the recall of control word pairs along with interference pairs. Barnes and Underwood detail the effects of $\mathrm{AB} / \mathrm{AC}$ memory paradigms on learning, focusing on how after learning List 2, there is gradual decrease in the frequency of List 1 responses during the recall of List 1 and 2 simultaneously (MMFR)—similar to an extinction curve [64]. This does not automatically signify that List 1 was "forgotten," but that List 2 may be causing interference and overpowering the recall of both responses. The control words in this paradigm help to address this issue [64].

1. Participants studied set 1 , which included eight noun-pairs, half containing interference pairs (AB). Following this study period, they completed a 20-s arithmetic distractor task.

2. Participants then completed Cued Recall 1, which involved the cued recall of Study Set 1 . Participants were exposed to the stem word (and a blank) and were required to recall its pair. Following this cued recall, they completed a 20-s arithmetic distractor task.

3. Participants then studied Set 2, which included eight noun-pairs, half of them containing interference pairs (AC) from Set 1, with the other half being new noun-pairs (FG). Following this study period, they completed a 20-s arithmetic distractor task.

4. Participants then completed Cued Recall 2, which involved the cued recall of Study Set 2. Participants were exposed to the stem word (and a blank) and were required to recall its pair. Following this cued recall, they completed a 20-s arithmetic distractor task.

5. Participants then completed a modified-modified free recall (MMFR), which involved the cued recall of Set 1 and Set 2. Since some of the words (A words) were paired with two different words (B and $C$ words), participants were instructed to recall both words, if they could recall both. From the MMFR cued recall test, Proactive Interference (PI) was calculated by subtracting the percentage of FG pairs recalled from the percentage of AC pairs recalled. From the MMFR cued recall test, Retroactive Interference (RI) was calculated by subtracting the percentage of DE pairs recalled from the percentage of $A B$ pairs recalled. Higher PI and RI scores are indicators of proactive and retroactive facilitation, and thus, correspond with decreased memory interference. That is, a lower PI and RI score indicate a greater (worse, unfavorable) memory interference effect.

For each Study Set (1 and 2), the noun-pairs were presented on the computer monitor screen for $5 \mathrm{~s}$ each. For each cued recall test (cued recall 1, cued recall 2, and MMFR), participants had $10 \mathrm{~s}$ to complete the recall. All noun-pairs had an imagery score of $\geq 6.0$. 
For each visit, participants completed a standard AB-DE AC-FG memory paradigm on a computer after the five-minute rest period following the exercise protocol. An example word list to evaluate this paradigm is shown in Table 1. Notably, separate word lists (of similar levels of imageability and concreteness) were employed for each visit. All of the word pair lists and recall lists were in a pseudorandomized order. As illustrated in Table 1, participants were exposed to List 1 (AB-DE), which consists of eight word pairs (e.g., Blouse-Nickel), completed a 20 second distractor task (i.e., mathematical problems), and then were exposed to the Cued Recall List 1 where they recalled the missing word from the word pair (e.g., Blouse____ _ ). Next, participants were exposed to List 2 (AC-FG), which also consisted of eight word pairs with some overlap of "A" words (e.g., Blouse-Bridge). After another 20-second distractor, participants were exposed to the cued recall of List 2 and attempted to recall the missing word from the word pairs. Lastly, a final 20-second distractor task was implemented and participants completed the modified-modified free recall (MMFR) word list where they recalled a combined list of List 1 and List 2 words. The MMFR consists of all the previously learned word pairs where some have only one missing word (DE, FG) and others have two missing word pair associations (AB, AC). MMFR was scored without regard to whether the response was from the first or second list. For each recall period (List 1, List 2, MMFR), there was only one trial and participants were not exposed to a familiarization trial before the experiment.

Multiple memory parameters were evaluated. The number of correctly recalled words from List 1 and List 2 were summed individually. Using the participant's MMFR results, the number of correctly recalled words from the subset of words $(\mathrm{AB}, \mathrm{DE}$ are within List 1 and $\mathrm{AC}, \mathrm{FG}$ are within List 2) was calculated. The percentage was also calculated by dividing the number of correct words by four, the total number of word pairs in the subset. Finally, proactive and retroactive interference were measured as a percentage. Using the cued recall results from List 1 and 2, proactive interference was measured by subtracting the percentage of correctly recalled FG pairs from the percentage of AC pairs (i.e., $\mathrm{AC}-\mathrm{FG}$ ) and RI by subtracting the percentage of correctly recalled $\mathrm{DE}$ pairs from the percentage of $\mathrm{AB}$ pairs (i.e., $\mathrm{AB}-\mathrm{DE})[65]$.

\section{Statistical Analysis}

All analyses were completed in SPSS (v. 23, IBM, SPSS, Armonk, NY, USA) or JASP (v. 0.9.1; Netherlands). Both frequentist and Bayesian analyses were computed. For the frequentist analyses, repeated measures analysis of variance (RM-ANOVA) was computed to examine the differences among memory outcomes between the control, moderate, and vigorous conditions. These frequentist analyses were supplemented with Bayesian RM-ANOVA analyses because Bayesian analyses do not assume large samples and typically smaller sample sizes can be analyzed without losing power while retaining precision [66,67]. For the Bayesian RM-ANOVA, Bayes Factors (BF) are reported, with a BF greater than 3.0 indicating substantial support for the alternative hypothesis [68]. For the frequentist analyses, statistical significance was set at an a priori alpha level of 0.05 .

\section{Results}

Descriptive statistics for the demographics of the sample are shown in Table 2. The sample was $68.4 \%$ female, with an average age of 20.32 years ( $S D=1.41$ years), average BMI of $24.25 \mathrm{~kg} / \mathrm{m}^{2}(\mathrm{SD}=$ $4.66 \mathrm{~kg} / \mathrm{m}^{2}$ ), and an average of 231.37 minutes/week (SD = 25.96 minutes/week) of exercise. 
Table 2. Descriptive statistics of the sample $(\mathrm{N}=19)$.

\begin{tabular}{ccc}
\hline Variable & Point Estimate & SD \\
\hline Gender, female (\%) & 68.4 & - \\
Age, mean years & 20.32 & 1.41 \\
Race, Non-Hispanic White $(\%)$ & 78.9 & - \\
Race, Non-Hispanic Black (\%) & 15.8 & - \\
Race, Mexican American (\%) & 5.3 & - \\
BMI, mean kg/m² & 24.25 & 4.66 \\
Average min/week of exercise & 231.37 & 25.96 \\
\hline
\end{tabular}

BMI, body mass index.

Physiological data across the experimental visits is provided in Table 3. The RM-ANOVA demonstrated that heart rate was significantly different at the vigorous-intensity level $(\mathrm{M}=172 \mathrm{bpm})$ compared to moderate $(\mathrm{M}=134 \mathrm{bpm})$ and control visits $(\mathrm{M}=69 \mathrm{bpm})(\mathrm{F}=802.35, p<0.0001$, $\left.\eta^{2} \mathrm{p}=0.97\right)$.

Table 3. Mean and standard deviation of heart rate across the experimental conditions.

\begin{tabular}{ccccccc}
\hline & \multicolumn{2}{c}{ Control $(\mathbf{n}=\mathbf{1 9})$} & Moderate, $\mathbf{5 0} \%$ HHR $(\mathbf{n}=\mathbf{1 9 )}$ & \multicolumn{2}{c}{ Vigorous, 80\% HHR (n= 19) } \\
\hline Variable & Mean & SD & Mean & SD & Mean & SD \\
\hline Heart rate at rest, bpm & 69.5 & 11.8 & 69.4 & 14.7 & 71.6 & 12.4 \\
Heart rate at 7.5 min, bpm & - & - & 134.6 & 8.0 & 172.9 & 7.2 \\
Heart rate at 15 min, bpm & - & - & 135.8 & 9.2 & 176.6 & 5.9 \\
Heart rate 5 min post, bpm & 72.3 & 12.4 & 84.0 & 14.2 & 91.9 & 16.8 \\
\hline
\end{tabular}

- signifies data was not assessed.

The frequentist and Bayesian RM-ANOVA results of the memory outcomes are displayed in Table 4 . The number of correctly recalled words from the cued recall of List 1 (AB-DE) was statistically significantly $\left(\mathrm{F}=4.63, p=0.01, \eta^{2} \mathrm{p}=0.205\right)$ higher for the vigorous-intensity condition $(\mathrm{M}=6.53, \mathrm{SD}=$ 1.54) as compared to the moderate $(\mathrm{M}=6.11, \mathrm{SD}=1.59)$ and control $(\mathrm{M}=5.0, \mathrm{SD}=2.56)$ conditions (Figure 2). When comparing these point estimates of the vigorous-intensity visit (6.53) to the control visit (5.0), this represents a $30.6 \%$ increase $((6.53-5.0 / 5.0) \times 100)$. When comparing the moderate-intensity visit (6.11) to the control visit (5.0), this represents a $22.2 \%$ increase $((6.11-5.0 / 5.0) \times 100)$. For these List 1 results, the Bayes Factor was 4.05, providing moderate support for the alternative hypothesis, in that the observed data are 4.05 times more likely under the alternative hypothesis than under the null hypothesis. There was no statistical significance found comparing the conditions across the number of correctly recalled words from the cued recall of List 2 (see Figure 3). Statistical significance was also observed for the respective number of correctly recalled AB words from the MMFR list $\left(\left(\mathrm{F}=4.97, \mathrm{p}=0.01, \eta^{2} \mathrm{p}=0.217\right.\right.$, Bayes Factor $\left.=4.94\right)$ and DE words from the MMFR list $(\mathrm{F}=4.72$, $p=0.01, \eta^{2} p=0.208$, Bayes Factor $\left.=3.76\right)$ ). No statistical significance was observed between proactive or retroactive interference across the experimental conditions. Differences between males and females were also examined, but there were no significant gender interaction effects for any of the outcomes. Although there was a statically significant difference between the conditions for the MMFR AB List $(\mathrm{F}=4.97, p=0.01)$, there was also evidence of a difference for both of the control lists, $\mathrm{DE}(\mathrm{F}=4.72$, $p=0.01)$ and FG $(\mathrm{F}=3.20, p=0.052)$. This aligns with our proactive and retroactive interference nonsignificant findings, as in order for proactive and retroactive interference to occur, there needs to be a change in the $\mathrm{AB}$ and $\mathrm{AC}$ interfering lists with no change in the $\mathrm{DE}$ and FG control lists. The acquired learning of List 1 may have also induced a proactive interference effect for the recall of List 2, which may explain why only the recall of List 1 was significant between the conditions. 
Table 4. RM-ANOVA results for memory outcome scores ${ }^{t}$ across the experimental conditions $(\mathrm{N}=19)$.

\begin{tabular}{ccccc}
\hline & Control & Moderate & Vigorous & \\
\cline { 1 - 3 } Variable & Mean (SD) & Mean (SD) & Mean (SD) & Results \\
\cline { 1 - 3 } \# Correct Cued Recall List 1 & $5.00(2.56)$ & $6.11(1.59)$ & $6.53(1.54)$ & $\mathrm{F}(2,36)=4.63, p=0.01^{*}, \eta^{2} \mathrm{p}=0.205, \mathrm{BF}=4.05$ \\
\# Correct Cued Recall List 2 & $5.31(1.94)$ & $6.00(1.73)$ & $5.47(2.14)$ & $\mathrm{F}(2,36)=1.71, p=0.19, \eta^{2} \mathrm{p}=0.087, \mathrm{BF}=0.47$ \\
\# Correct MMFR AB List & $2.10(1.24)$ & $2.47(1.21)$ & $3.05(0.97)$ & $\mathrm{F}(2,36)=4.97, p=0.01^{*}, \eta^{2} \mathrm{p}=0.217, \mathrm{BF}=4.94$ \\
\# Correct MMFR DE List & $2.10(1.44)$ & $2.36(1.21)$ & $3.00(1.15)$ & $\mathrm{F}(2,36)=4.72, p=0.01^{*}, \eta^{2} \mathrm{p}=0.208, \mathrm{BF}=3.76$ \\
\# Correct MMFR AC List & $2.21(1.22)$ & $2.57(1.21)$ & $2.57(1.30)$ & $\mathrm{F}(2,36)=0.795, p=0.46, \eta^{2} \mathrm{p}=0.042, \mathrm{BF}=0.25$ \\
\# Correct MMFR FG List & $2.63(1.16)$ & $3.26(0.87)$ & $2.84(1.25)$ & $\mathrm{F}(2,36)=3.20, p=0.052, \eta^{2} \mathrm{p}=0.151, \mathrm{BF}=0.51$ \\
Proactive Interference & $-11.84(33.71)$ & $-14.47(24.03)$ & $-6.58(28.67)$ & $\mathrm{F}(2,36)=0.411, p=0.66, \eta^{2} \mathrm{p}=0.022, \mathrm{BF}=0.82$ \\
Retroactive Interference & $0.00(25.36)$ & $-1.32(26.96)$ & $1.32(24.25)$ & $\mathrm{F}(2,36)=0.062, p=0.94, \eta^{2} \mathrm{p}=0.003, \mathrm{BF}=0.14$ \\
\hline
\end{tabular}

${ }^{t}$ Memory outcome scores are calculated as follows $(\mathrm{N}=19)$. Proactive interference (PI) and retroactive interference (RI) are measured as a percentage. Using the cued recall results from List 1 and 2, PI is measured by subtracting the percentage of correctly recalled FG pairs from the percentage of AC pairs (e.g., AC-FG) and RI by subtracting the percentage of correctly recalled $\mathrm{DE}$ pairs from the percentage of $\mathrm{AB}$ pairs (e.g., $\mathrm{AB}-\mathrm{DE}$ ). For each outcome, a RM-ANOVA was computed. The F value represents the main effect for condition. BF, Bayes Factor; $\eta^{2} p=$ Partial-eta squared.

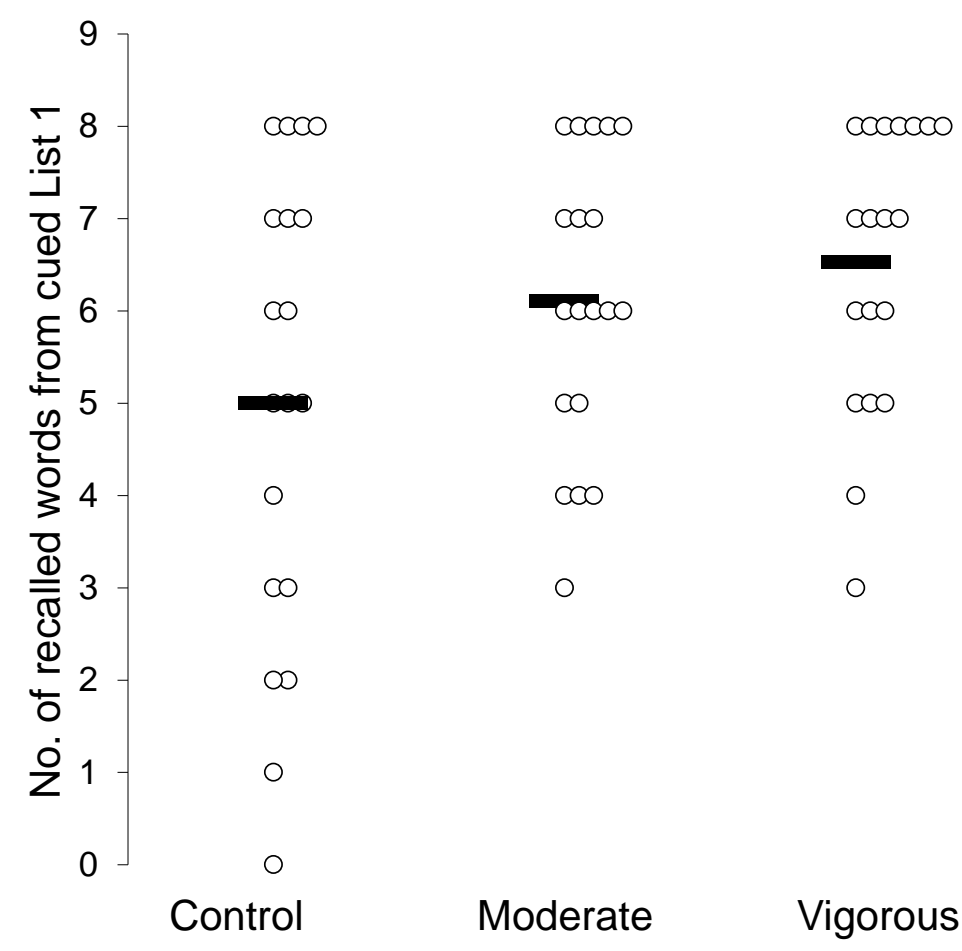

Figure 2. Individual level data $(n=19)$ for the number of correctly recalled words from the cued recall of List 1 across the experimental conditions (control, moderate-intensity, and vigorous-intensity exercise). Means are represented by the black bar: control $(M=5.0)$, moderate $(M=6.11)$, and vigorous ( $\mathrm{M}=6.53)$. 


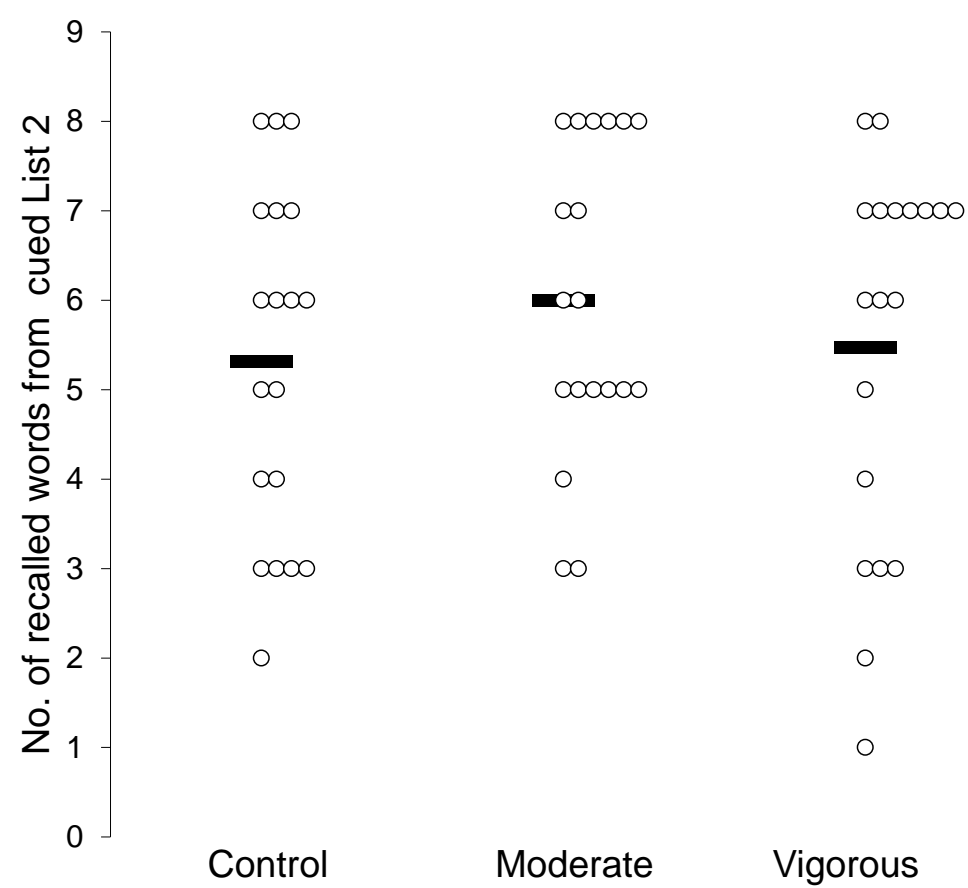

Figure 3. Individual level data $(n=19)$ for the number of correctly recalled words from the cued recall of List 2 across the experimental conditions (control, moderate-intensity, and vigorous-intensity exercise). Means are represented by the black bar: control $(M=5.32)$, moderate $(M=6.0)$, and vigorous $(\mathrm{M}=5.47)$.

Brinley plots of the control versus moderate visit and control versus vigorous visit were created for the cued recall of List 1, proactive interference, and retroactive interference (see Figures 4-9). There is a line of no change imposed across each graph and if the data points fall on the line, this signifies that there was no change from the control to the moderate/vigorous visit. If the data points are above the line, this indicates that the participant performed better for the moderate/vigorous visit when compared to the control visit. Figures 4 and 5, the results from the cued recall of list 1 , indicate that participants performed better during the exercise visits compared to the control visit. For proactive interference, Figures 6 and 7 show mixed results for both moderate and vigorous conditions. Finally, for retroactive interference, Figures 8 and 9 show similar results with no effect of acute exercise on attenuating a retroactive interference effect.

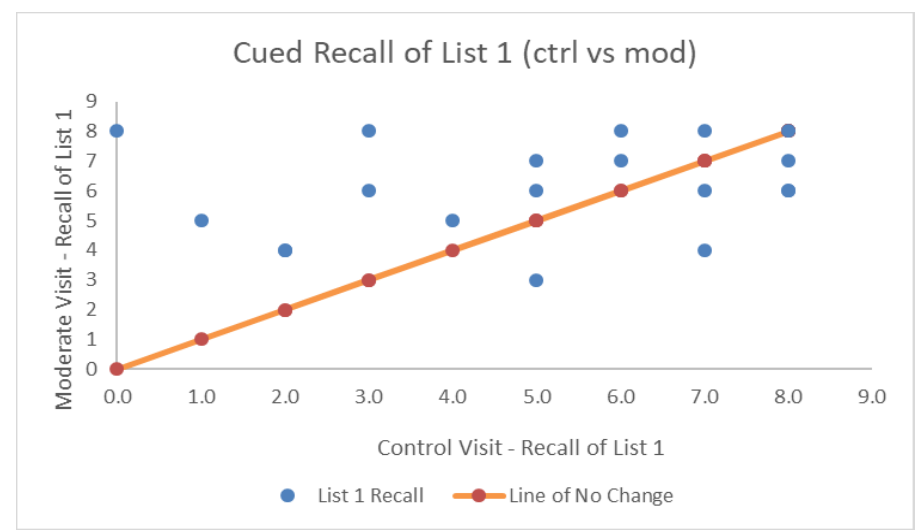

Figure 4. Cued recall of List $1-$ Control visit versus moderate visit. Each data point represents one participant's performance across the two conditions. Data points on or below the line of no change signify no beneficial effect of the exercise condition when compared to the control visit. There was no statistically significant difference between the moderate-intensity visit and control visit on the cued recall of list 1. 


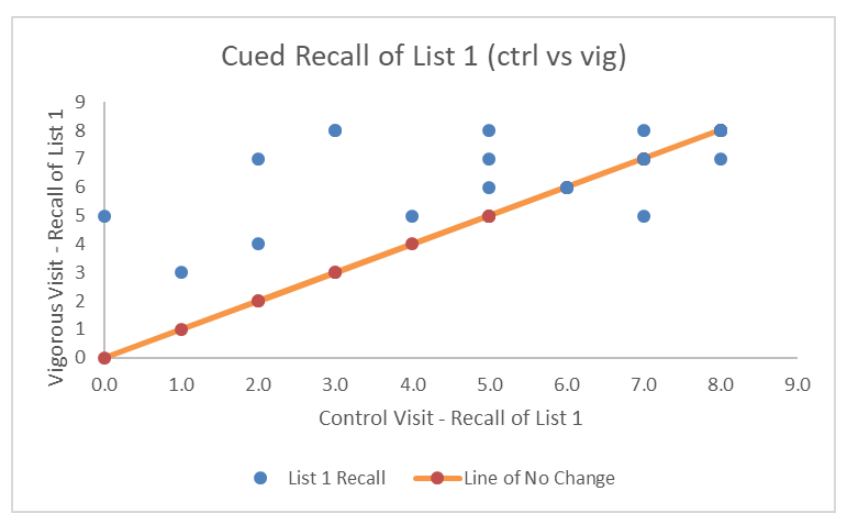

Figure 5. Cued recall of List 1-Control visit versus vigorous visit. Each data point represents one participant's performance across the two conditions. Data points on or below the line of no change signify no beneficial effect of the exercise condition compared to the control visit. In this case, there was a statistically significant difference in memory performance between the vigorous-intensity visit and the control visit, with memory performance increasing in the visit with a bout of vigorous exercise.

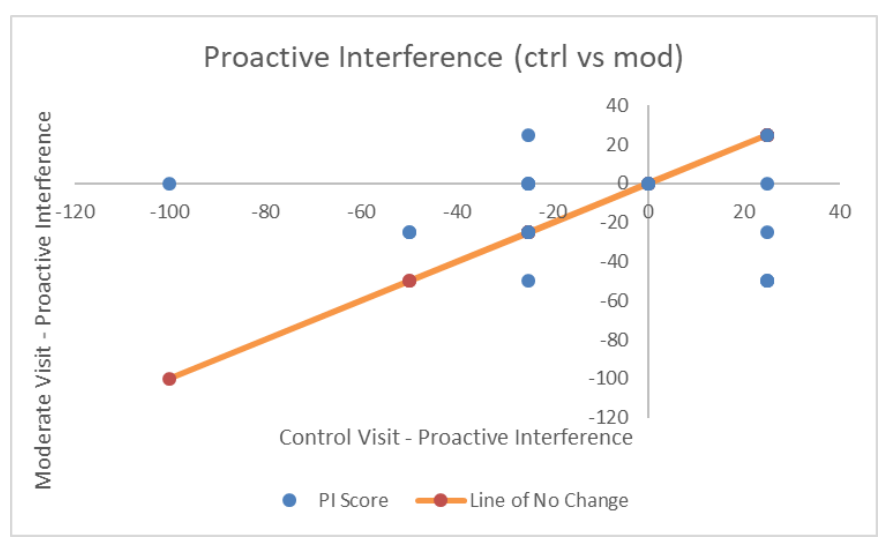

Figure 6. Proactive interference-Control visit versus moderate visit. Each data point represents one participant's performance across the two conditions. Data points on or below the line of no change signify no beneficial effect of the exercise condition compared to control at increasing memory performance. There was no statistically significant difference between the moderate-intensity visit and control visit on proactive interference.

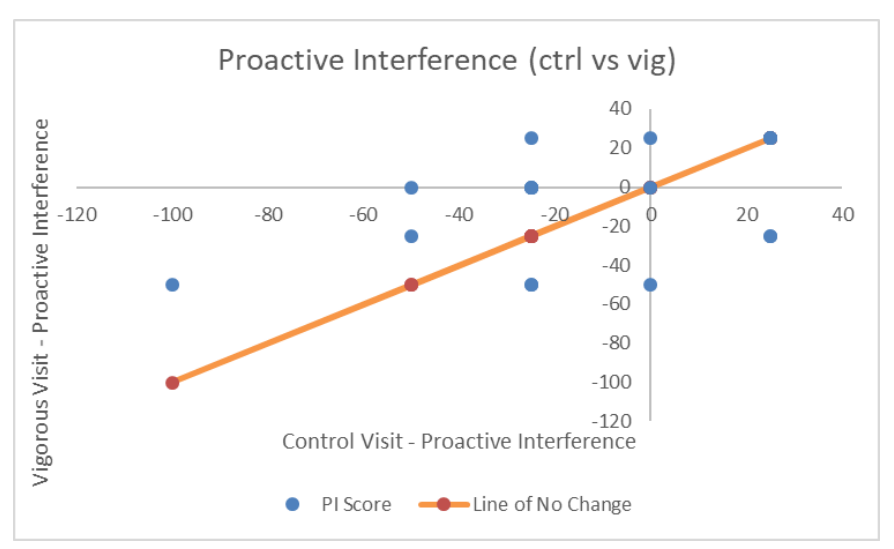

Figure 7. Proactive interference-Control visit versus vigorous visit. Each data point represents one participant's performance across the two conditions. Data points on or below the line of no change signify no beneficial effect of the exercise condition when compared to the control visit. There was no statistically significant difference between the vigorous-intensity visit and control visit on proactive interference. 


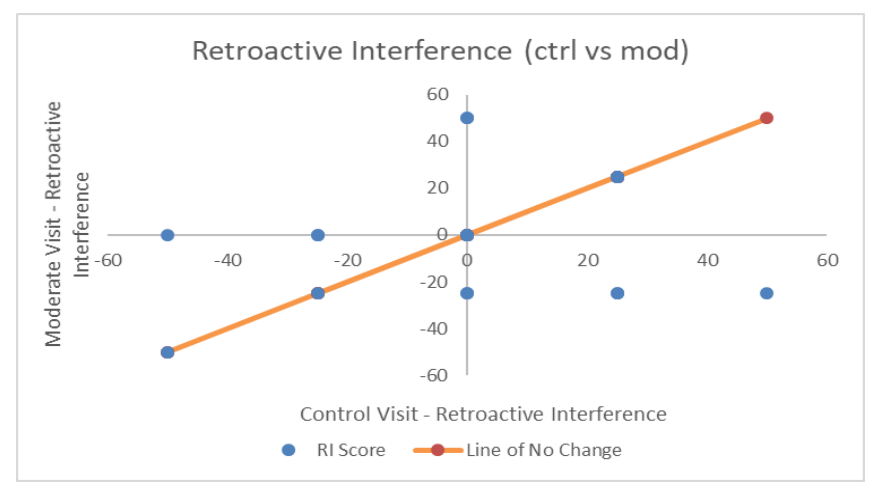

Figure 8. Retroactive interference-Control visit versus moderate visit. Each data point represents one participant's performance across the two conditions. Data points on or below the line of no change signify no beneficial effect of the exercise condition when compared to the control visit. There was no statistically significant difference between the moderate-intensity visit and control visit on retroactive interference.

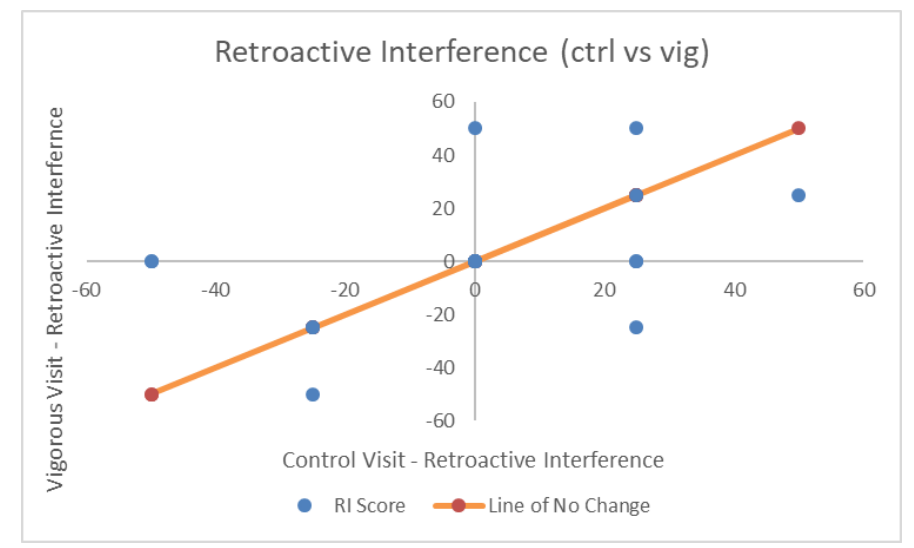

Figure 9. Retroactive interference-Control visit versus vigorous visit. Each data point represents one participant's performance across the two conditions. Data points on or below the line of no change signify no beneficial effect of the exercise condition when compared to the control visit. There was no statistically significant difference between the vigorous-intensity visit and control visit on retroactive interference.

\section{Discussion}

As we have discussed previously [20], few studies have examined the effects of multiple exercise intensities on short-term, paired-associative memory function in a within-subject design, and there is also limited research on the effect of exercise on memory interference. To address these gaps in the literature, our study implemented a within-subject design to examine the potential intensity-specific effects of acute exercise on paired-associative memory and memory interference. Previous literature demonstrates that acute exercise can enhance memory function $[6,10,15,20,21,38,69]$. Our findings align with these previous experiments. Our results also align with a recent review suggesting that there may be an intensity-specific effect of acute exercise on memory function [20]. Adding to this body of work, in our experiment, we also investigated the effects of acute exercise on memory interference.

In the present study, exercise intensity influenced memory function, with the vigorous-intensity protocol having the most significant increase in the number of correctly recalled words for the cued recall of List 1. Interestingly, however, memory performance was not significantly increased for the cued recall of List 2, which may be due to a proactive interference effect of List 1 on List 2. As stated previously, the increase in List 1 outcomes may be a result of a potential intensity-specific effect of acute exercise on LTP [21]. Another potential avenue through which exercise may influence memory is through arousal and psychologically-based attention effects [21]. Interestingly, in the 
present experiment, the exercise conditions, as seen in Figure 2, demonstrated a reduction of low performance scores for the recall of List 1 . This suggests that the beneficial effects of acute exercise on paired-associative memory may, perhaps, contribute by attenuating forgetting. Future work is needed to evaluate whether acute exercise improves long-term memory via enhancing memory and/or attenuating forgetting [70].

Although the literature on exercise and memory interference is sparse, it has been demonstrated that acute exercise may enhance working memory, suggesting that it may also play a role in attenuating memory interference [20]. To date, only three published studies have examined the effects of acute exercise on cognitive-related (not procedural) memory interference. These three studies, conducted in our laboratory, are briefly discussed as follows. Haynes and Loprinzi demonstrated that memory recall was improved when moderate-intensity exercise occurred prior to the memory task (encoding), although acute exercise did not statistically significantly attenuate proactive memory interference [14]. Wingate et al. established that moderate-intensity acute exercise may have a protective effect against retroactive, but not proactive memory interference [56]. In a separate study examining the role of moderate- and vigorous-intensity acute exercise before memory encoding, a control condition with no exercise, exercise during memory encoding, and exercise after memory encoding, the group that exercised prior to memory encoding recalled the most words from List B of the Rey Auditory Verbal Learning memory task, though differences between the conditions were not statistically significant for moderate- or vigorous-intensity acute exercise [13].

Our present study expands upon this emerging literature by manipulating exercise intensity in a within-subject design and, unlike the previous studies on exercise and memory interference $[13,14,56]$, the present study more robustly assessed both proactive and retroactive interference. Our experiment did not provide convincing evidence for an effect of acute exercise to attenuate memory interference. Before dismissing a potential effect of acute exercise on attenuating memory interference, further studies need to be completed and additional explanations need to be considered. The current study may be limited by its small sample size. It is possible that the participants experienced a familiarization effect from being repeatedly tested on the paired-associative task, but as our visits were counterbalanced, such an effect is unlikely to have influenced the between-visit results. The duration of the exercise bout may need to be longer to be matched for energy expenditure (longer duration for less intensive exercise). There may also be an important distinction between the cognitive benefits of open- and closed-skill exercise. Closed-skill exercise signifies an aspect of predictability (e.g., running on a treadmill), whereas open-skill activities may be unpredictable (e.g., playing racquetball) [32]. When compared experimentally, an open-skill exercise condition demonstrated more efficient neuronal resource allocation and higher levels of exercise-induced BDNF levels [32], which may have implications for attenuating memory interference, given that BDNF is a key neurotrophin for the persistence of long-term memory [71]. Future studies may wish to consider conducting a within-subject design involving a control condition, open-skill bout of exercise, and closed-skill bout of exercise along with the completion of a memory interference task. This may help to determine whether there is a differential effect of open or closed-skill exercise on the attenuation of memory interference. Such work should also consider implementing a delayed recall of the MMFR list to better evaluate long-term memory. Evaluating the test-retest reliability of our evaluated memory interference task as well as other memory interference protocols would also increase the strength of future research.

In conclusion, this study implemented a within-subject design to examine the effects of acute exercise intensity on paired-associative memory and the attenuation of memory interference. The results demonstrated that moderate- and vigorous-intensity acute exercise did not have an effect on memory interference, which aligns with other recent experiments $[13,14,56]$. However, our results provide support for vigorous exercise having a greater effect on enhancing paired-associative memory. Given the paucity of research in this area, future work evaluating the potential role of acute exercise on memory interference is warranted. 
Author Contributions: L.C. and P.L. were involved in conceptualizing the study. L.C. collected the data and drafted the manuscript. L.C. and P.L. were involved in the statistical analyses and revising the manuscript.

Funding: This research received no external funding.

Conflicts of Interest: The authors declare no conflict of interest.

\section{References}

1. Frith, E.; Addoh, O.; Mann, J.R.; Windham, B.G.; Loprinzi, P.D. Individual and Combined Associations of Cognitive and Mobility Limitations on Mortality Risk in Older Adults. Mayo. Clin. proc. 2017, 92, 1494-1501. [CrossRef]

2. Bird, C.M.; Burgess, N. The hippocampus and memory: Insights from spatial processing. Nat. rev. Neurosci. 2008, 9, 182-194. [CrossRef]

3. Weilbacher, R.A.; Gluth, S. The Interplay of Hippocampus and Ventromedial Prefrontal Cortex in Memory-Based Decision Making. Brain sci. 2016, 7, 4. [CrossRef] [PubMed]

4. Tulving, E. Elements of Episodic Memory; Oxford University Press: Oxford, UK, 1983.

5. Baddeley, A. Working memory and language: An overview. J. Commun. Disord. 2003, 36, 189-208. [CrossRef]

6. Warburton, D.E.R.; Bredin, S.S.D. Health benefits of physical activity: A systematic review of current systematic reviews. Curr. Opin. Cardiol. 2017, 32, 541-556. [CrossRef]

7. Loprinzi, P.D.; Herod, S.M.; Cardinal, B.J.; Noakes, T.D. Physical activity and the brain: A review of this dynamic, bi-directional relationship. Brain Res. 2013, 1539, 95-104. [CrossRef] [PubMed]

8. Ponce, P.; Loprinzi, P.D. A bi-directional model of exercise and episodic memory function. Med. Hypotheses 2018, 117, 3-6. [CrossRef]

9. Sng, E.; Frith, E.; Loprinzi, P.D. Temporal Effects of Acute Walking Exercise on Learning and Memory Function. Am. J. Health Promot. 2017. [CrossRef] [PubMed]

10. Loprinzi, P.D.; Edwards, M.K.; Frith, E. Potential avenues for exercise to activate episodic memory-related pathways: A narrative review. Eur. J. Neurosci. 2017, 46, 2067-2077. [CrossRef] [PubMed]

11. Siddiqui, A.; Loprinzi, P.D. Experimental Investigation of the Time Course Effects of Acute Exercise on False Episodic Memory. J. Clin. Med. 2018, 7, 157. [CrossRef]

12. Haynes Iv, J.T.; Frith, E.; Sng, E.; Loprinzi, P.D. Experimental Effects of Acute Exercise on Episodic Memory Function: Considerations for the Timing of Exercise. Psychol. Rep. 2018. [CrossRef] [PubMed]

13. Frith, E.; Sng, E.; Loprinzi, P. Randomized Controlled Trial Considering Varied Exercises for Reducing Proactive Memory Interference. J. Clin. Med. 2018, 7, 147. [CrossRef] [PubMed]

14. Haynes, J.; Loprinzi, P. Acute cardiovascular exercise on proactive memory interference. J. Cogn. Enhanc. 2018, 3, 139-143. [CrossRef]

15. Chang, Y.K.; Labban, J.D.; Gapin, J.I.; Etnier, J.L. The effects of acute exercise on cognitive performance: A meta-analysis. Brain Res. 2012, 1453, 87-101. [CrossRef] [PubMed]

16. Lambourne, K.; Tomporowski, P. The effect of exercise-induced arousal on cognitive task performance: A meta-regression analysis. Brain Res. 2010, 1341, 12-24. [CrossRef]

17. McMorris, T.; Hale, B.J. Differential effects of differing intensities of acute exercise on speed and accuracy of cognition: a meta-analytical investigation. Brain Cogn. 2012, 80, 338-351. [CrossRef]

18. Roig, M.; Nordbrandt, S.; Geertsen, S.S.; Nielsen, J.B. The effects of cardiovascular exercise on human memory: A review with meta-analysis. Neurosci. Biobehav. Rev. 2013, 37, 1645-1666. [CrossRef] [PubMed]

19. McMorris, T.; Sproule, J.; Turner, A.; Hale, B.J. Acute, intermediate intensity exercise, and speed and accuracy in working memory tasks: A meta-analytical comparison of effects. Physiol. Behav. 2011, 102, 421-428. [CrossRef]

20. Loprinzi, P.D. Intensity-specific effects of acute exercise on human memory function: Considerations for the timing of exercise and the type of memory. Health Promot. Perspect. 2018, 8, 255-262. [CrossRef]

21. Loprinzi, P.D.; Ponce, P.; Frith, E. Hypothesized mechanisms through which acute exercise influences episodic memory. Physiol. Int. 2018, 105, 285-297. [CrossRef]

22. Poo, M.M.; Pignatelli, M.; Ryan, T.J.; Tonegawa, S.; Bonhoeffer, T.; Martin, K.C.; Rudenko, A.; Tsai, L.H.; Tsien, R.W.; Fishell, G.; et al. What is memory? The present state of the engram. BMC Biol. 2016, 14, 40. [CrossRef] 
23. Audiffren, M. Acute exercise and psychological functions: A cognitive-energetic approach. Exerc. Cogn. Funct. 2009, 3-39. [CrossRef]

24. Noble, E.E.; Mavanji, V.; Little, M.R.; Billington, C.J.; Kotz, C.M.; Wang, C. Exercise reduces diet-induced cognitive decline and increases hippocampal brain-derived neurotrophic factor in CA3 neurons. Neurobiol. Learn. Mem. 2014, 114, 40-50. [CrossRef] [PubMed]

25. Davis, R.L.; Zhong, Y. The Biology of Forgetting-A Perspective. Neuron 2017, 95, 490-503. [CrossRef] [PubMed]

26. Osgood, C.E. An investigation into the causes of retroactive interference. J. Exp. Psychol. 1948, 38, $132-154$. [CrossRef] [PubMed]

27. Shimamura, A.P.; Jurica, P.J.; Mangels, J.A.; Gershberg, F.B.; Knight, R.T. Susceptibility to Memory Interference Effects following Frontal Lobe Damage: Findings from Tests of Paired-Associate Learning. J. Cogn. Neurosci. 1995, 7, 144-152. [CrossRef]

28. Bridge, D.J. Memory \& Cognition: What Difference Does Gender Make? Available online: https://surface.syr. edu/honors_capstone/655/ (accessed on 25 May 2019).

29. Erickson, K.I.; Leckie, R.L.; Weinstein, A.M. Physical activity, fitness, and gray matter volume. Neurobiol. Aging 2014, 35. [CrossRef]

30. Samrani, G.; Bäckman, L.; Persson, J. Age-differences in the temporal properties of proactive interference in working memory. Psychol. Aging 2017, 32, 722-731. [CrossRef] [PubMed]

31. Bennett, R. Proactive interference in short-term memory: Fundamental forgetting processes. J. Verbal Learn. Verbal Behav. 1975, 123-144. [CrossRef]

32. Hung, C.L.; Tseng, J.W.; Chao, H.H.; Hung, T.M.; Wang, H.S. Effect of Acute Exercise Mode on Serum Brain-Derived Neurotrophic Factor (BDNF) and Task Switching Performance. J. Clin. Med. 2018, 7, 301. [CrossRef]

33. Siddiqui, S.V.; Chatterjee, U.; Kumar, D.; Siddiqui, A.; Goyal, N. Neuropsychology of prefrontal cortex. Indian J. Psychiatry 2008, 50, 202-208.

34. Guise, K.G.; Shapiro, M.L. Medial Prefrontal Cortex Reduces Memory Interference by Modifying Hippocampal Encoding. Neuron 2017, 94, 183-192. [CrossRef]

35. Ahmed, O.J.; Mehta, M.R. Running speed alters the frequency of hippocampal gamma oscillations. J. Neurosci. 2012, 32, 7373-7383. [CrossRef]

36. Zheng, J.; Stevenson, R.F.; Mander, B.A.; Mnatsakanyan, L.; Hsu, F.P.K.; Vadera, S.; Knight, R.T.; Yassa, M.A.; Lin, J.J. Multiplexing of Theta and Alpha Rhythms in the Amygdala-Hippocampal Circuit Supports Pattern Separation of Emotional Information. Neuron 2019, 102, 887-898. [CrossRef] [PubMed]

37. Thomas, A.G.; Dennis, A.; Bandettini, P.A.; Johansen-Berg, H. The effects of aerobic activity on brain structure. Front. Psychol. 2012. [CrossRef] [PubMed]

38. Loprinzi, P.D.; Frith, E.; Edwards, M.K.; Sng, E.; Ashpole, N. The Effects of Exercise on Memory Function Among Young to Middle-Aged Adults: Systematic Review and Recommendations for Future Research. Am. J. Health Promot. 2018, 32, 691-704. [CrossRef] [PubMed]

39. Sudo, M.; Komiyama, T.; Aoyagi, R.; Nagamatsu, T.; Higaki, Y.; Ando, S. Executive function after exhaustive exercise. Eur. J. Appl. Physiol. 2017, 117, 2029-2038. [CrossRef] [PubMed]

40. Endo, K.; Matsukawa, K.; Liang, N.; Nakatsuka, C.; Tsuchimochi, H.; Okamura, H.; Hamaoka, T. Dynamic exercise improves cognitive function in association with increased prefrontal oxygenation. J. Physiol. Sci. 2013, 63, 287-298. [CrossRef]

41. McNerney, M.W.; Radvansky, G.A. Mind racing: The influence of exercise on long-term memory consolidation. Memory 2015, 23, 1140-1151. [CrossRef]

42. Delancey, D.; Frith, E.; Sng, E.; Loprinzi, P.D. Randomized controlled trial examining the long-term memory effects of acute exercise during the memory consolidation stage of memory. J. Cogn. Enhance. 2018, 1-6. [CrossRef]

43. Frith, E.; Sng, E.; Loprinzi, P.D. Randomized controlled trial evaluating the temporal effects of high-intensity exercise on learning, short-term and long-term memory, and prospective memory. Eur. J. Neurosci. 2017, 46, 2557-2564. [CrossRef]

44. Sng, E.; Frith, E.; Loprinzi, P.D. Temporal Effects of Acute Walking Exercise on Learning and Memory Function. Am. J. Health Promot. 2018, 32, 1518-1525. [CrossRef] [PubMed] 
45. Jubelt, L.E.; Barr, R.S.; Goff, D.C.; Logvinenko, T.; Weiss, A.P.; Evins, A.E. Effects of transdermal nicotine on episodic memory in non-smokers with and without schizophrenia. Psychopharmacology (Berl) 2008, 199, 89-98. [CrossRef] [PubMed]

46. Klaming, R.; Annese, J.; Veltman, D.J.; Comijs, H.C. Episodic memory function is affected by lifestyle factors: A 14-year follow-up study in an elderly population. Neuropsychol. Dev. Cogn. Sect. B Aging Neuropsychol. Cogn. 2017, 24, 528-542. [CrossRef]

47. Henry, J.D.; Rendell, P.G. A review of the impact of pregnancy on memory function. J. Clin. Exp. Neuropsychol. 2007, 29, 793-803. [CrossRef]

48. Labban, J.D.; Etnier, J.L. Effects of acute exercise on long-term memory. Res. Q. Exerc. Sport 2011, 82, 712-721. [CrossRef]

49. Sherman, S.M.; Buckley, T.P.; Baena, E.; Ryan, L. Caffeine Enhances Memory Performance in Young Adults during Their Non-optimal Time of Day. Front. Psychol. 2016, 7, 1764. [CrossRef] [PubMed]

50. Wammes, J.D.; Good, T.J.; Fernandes, M.A. Autobiographical and episodic memory deficits in mild traumatic brain injury. Brain Cogn. 2017, 111, 112-126. [CrossRef]

51. Ilieva, I.P.; Hook, C.J.; Farah, M.J. Prescription Stimulants' Effects on Healthy Inhibitory Control, Working Memory, and Episodic Memory: A Meta-analysis. J. Cogn. Neurosci. 2015, 27, 1069-1089. [CrossRef] [PubMed]

52. Hindocha, C.; Freeman, T.P.; Xia, J.X.; Shaban, N.D.C.; Curran, H.V. Acute memory and psychotomimetic effects of cannabis and tobacco both 'joint' and individually: A placebo-controlled trial. Psychol. Med. 2017, 47, 2708-2719. [CrossRef]

53. Le Berre, A.P.; Fama, R.; Sullivan, E.V. Executive Functions, Memory, and Social Cognitive Deficits and Recovery in Chronic Alcoholism: A Critical Review to Inform Future Research. Alcohol. Clin. Exp. Res. 2017, 41, 1432-1443. [CrossRef] [PubMed]

54. Loprinzi, P.D.; Edwards, M.K.; Frith, E. Exercise and Prospective Memory. J. Lifestyle Med. 2018, 8, 51-59. [CrossRef]

55. Wade, B.; Loprinzi, P.D. The Experimental Effects of Acute Exercise on Long-Term Emotional Memory. J. Clin. Med. 2018, 7, 486. [CrossRef]

56. Wingate, S.; Crawford, L.; Frith, E.; Loprinzi, P.D. Experimental investigation of the effects of acute exercise on memory interference. Health Promot. Perspect. 2018, 8, 208-214. [CrossRef] [PubMed]

57. Clinic, M. Exercise Intensity: How to Measure It. Available online: https://www.mayoclinic.org/healthylifestyle/fitness/in-depth/exercise-intensity/art-20046887 (accessed on 15 February 2019).

58. Garber, C.E.; Blissmer, B.; Deschenes, M.R.; Franklin, B.A.; Lamonte, M.J.; Lee, I.M.; Nieman, D.C.; Swain, D.P. Quantity and quality of exercise for developing and maintaining cardiorespiratory, musculoskeletal, and neuromotor fitness in apparently healthy adults: Guidance for prescribing exercise. Med. Sci. Sports Exerc. 2011, 43, 1334-1359. [CrossRef]

59. Litt, R.A.; Wang, H.C.; Sailah, J.; Badcock, N.A.; Castles, A. Paired associate learning deficits in poor readers: The contribution of phonological input and output processes. Q. J. Exp. Psychol. (Hove) 2019, 72, 616-633. [CrossRef] [PubMed]

60. Elvevåg, B.; Egan, M.F.; Goldberg, T.E. Paired-associate learning and memory interference in schizophrenia. Neuropsychologia 2000, 38, 1565-1575. [CrossRef]

61. Iodice, R.; Meilan, J.J.G.; Ramos, J.C.; Small, J.A. Sentence Context and Word-Picture Cued-Recall Paired-Associate Learning Procedure Boosts Recall in Normal and Mild Alzheimer's Disease Patients. Behav. Neurol. 2018, 2018, 7401465. [CrossRef]

62. Ebert, P.L.; Anderson, N.D. Proactive and retroactive interference in young adults, healthy older adults, and older adults with amnestic mild cognitive impairment. J. Int. Neuropsychol. Soc. 2009, 15, 83-93. [CrossRef]

63. Curiel, R.E.; Crocco, E.; Acevedo, A.; Duara, R.; Agron, J.; Loewenstein, D.A. A New Scale for the Evaluation of Proactive and Retroactive Interference in Mild Cognitive Impairment and Early Alzheimer's Disease. J. Aging Sci. 2013, 1. [CrossRef]

64. Barnes, J.M.; Underwood, B.J. Fate of first-list associations in transfer theory. J. Exp. Psychol. 1959, 58, 97-105. [CrossRef] [PubMed]

65. Burton, R.L.; Lek, I.; Caplan, J.B. Associative independence revisited: Competition between conflicting associations can be resolved or even reversed in one trial. Q. J. Exp. Psychol. (Hove) 2017, 70, 832-857. [CrossRef] 
66. Lee, S.Y.; Song, X.Y. Evaluation of the Bayesian and Maximum Likelihood Approaches in Analyzing Structural Equation Models with Small Sample Sizes. Multivar. Behav. Res. 2004, 39, 653-686. [CrossRef] [PubMed]

67. Hox, J.; Van de Schoot, R.; Matthijsse, S. How few countries will do? Comparative survey analysis from a Bayesian perspective. Surv. Res. Methods 2012, 6, 87-93.

68. Jarosz, A.F.; Wiley, J. What Are the Odds? A Practical Guide to Computing and Reporting Bayes Factors. J. Probl. Solving 2014, 7, 1932-6246. [CrossRef]

69. Mandolesi, L.; Polverino, A.; Montuori, S.; Foti, F.; Ferraioli, G.; Sorrentino, P.; Sorrentino, G. Effects of Physical Exercise on Cognitive Functioning and Wellbeing: Biological and Psychological Benefits. Front. Psychol. 2018, 9, 509. [CrossRef]

70. Ferguson, L.; Cantrelle, J.; Loprinzi, P.D. Experimental effects of exercise on forgetting. OBM Integr. Complement. Med. 2018, 3, 034. [CrossRef]

71. Loprinzi, P.D.; Frith, E. A brief primer on the mediational role of BDNF in the exercise-memory link. Clin. Physiol. Funct. Imaging 2018. [CrossRef]

(C) 2019 by the authors. Licensee MDPI, Basel, Switzerland. This article is an open access article distributed under the terms and conditions of the Creative Commons Attribution (CC BY) license (http://creativecommons.org/licenses/by/4.0/). 